\section{A produção pública de medicamentos no Brasil: uma visão geral}

\author{
Public production of medicines \\ in Brazil: an overview
}

\author{
1 Escola Nacional de Saúde \\ Pública Sergio Arouca, \\ Fundação Oswaldo Cruz, \\ Rio de Janeiro, Brasil. \\ 2 Organização \\ Pan-Americana da Saúde, \\ Washington DC, \\ Estados Unidos. \\ Correspondência \\ E. A. Oliveira \\ Núcleo de Assistência \\ Farmacêutica, Escola \\ Nacional de Saúde \\ Pública Sergio Arouca, \\ Fundação Oswaldo Cruz. \\ Av. Brasil 4036, \\ Rio de Janeiro, $R J$ \\ 21040-361, Brasil. \\ biaol@ensp.fiocruz.br
}

\begin{abstract}
This paper analyzes the role of government production of medicines in Brazil, based on a review of the literature and public documents. It begins by examining the main characteristics of the national and international pharmaceutical industry and market dimensions, mainly in the public sector. The government view was examined through Ministry of Health documents. The article concludes with a diagnosis of the state-owned pharmaceutical industry in Brazil, showing a variety of difficulties faced by the laboratories due to political and institutional constraints, management deficiencies, shortage of qualified human resources, and low technological capability.
\end{abstract}

Pharmaceutical Preparations; National Drug Policy; Official Laboratory
Egléubia Andrade de Oliveira 1

Maria Eliana Labra ${ }^{1}$

Jorge Bermudez ${ }^{2}$

\section{Introdução}

O medicamento não é um produto qualquer: ele pode aliviar a dor e salvar vidas. É também um produto singular, dado que envolve diferentes fases no processo de produção e comercialização. Para que esse produto chegue ao consumidor, é necessário realizar pesquisas de moléculas biologicamente ativas, desenvolver o processo de formulação com vistas à produção em escala industrial e alcançar o processamento final. A partir de então, principia o processo de comercialização e distribuição na rede comercial e nos serviços de saúde 1,2,3. Além disso, o medicamento possui características mercadológicas diferentes de outros produtos manufaturados, pois integra um mercado em que praticamente não existe concorrência de escala entre os produtores. A especificidade da concorrência no setor farmacêutico baseia-se na diferenciação do produto calcada no investimento continuado e de grande porte em atividade de pesquisa e desenvolvimento (P\&D) e de marketing 4 .

A indústria farmacêutica pode ser descrita como um conjunto de oligopólios com multiprodutos diferenciados em segmentos de classes terapêuticas específicas, cujo consumo é fortemente mediado pela necessidade de prescrição médica. A diferenciação dos produtos por classe terapêutica, princípio ativo, composição química e embalagens gera um leque va- 
riado de apresentações direcionadas ao consumidor, dificultando a escolha do produto de forma racional 4. É, portanto, um setor baseado em ciência, cuja principal fonte de inovação e diferenciação dos produtos resulta de novos conhecimentos gerados a partir da infra-estrutura de ciência e tecnologia (C\&T) e das atividades de P\&D das empresas 1,4 .

Nos últimos anos, nos países em desenvolvimento, as questões dos medicamentos e da assistência farmacêutica vêm ganhando espaço na agenda governamental e na sociedade. Algumas razões podem justificar o incremento dessa visibilidade. O desenvolvimento tecnológico é uma delas. Nas últimas décadas, tem-se ampliado a abrangência do medicamento, de modo que, hoje, existem produtos para quase todas as enfermidades. Além disso, os medicamentos são cada vez mais seguros e eficazes, contribuindo para aumentar a expectativa e qualidade de vida. Por tudo isso, são apontados como um importante indicador de qualidade dos serviços de saúde. De fato, o abastecimento regular de medicamentos na rede pública é considerado um dos elementos que interferem na efetividade de um programa de saúde 5 . Ao contrário, um abastecimento irregular pode promover a desmotivação dos profissionais e o descontentamento da população.

Embora o medicamento tenha se tornado imprescindível para a sociedade e o Estado, sua produção tem sido capturada por poucas empresas líderes. De grande porte, atuam de forma globalizada em segmentos específicos (classes terapêuticas), mediante estratégias de diferenciação de produtos, num processo iniciado nos anos 80 com a fusão dos maiores laboratórios do mundo. O objetivo dessa estratégia era aumentar a rentabilidade e fazer investimentos de maior porte. De fato, dados apontam que as dez maiores indústrias farmacêuticas do mundo respondem por cerca de $40,4 \%$ do mercado mundial 6 . A concentração em grandes mercados com a participação de número reduzido de empresas é uma das principais características do mercado farmacêutico internacional de nossos dias. Nos anos 90, as fusões continuaram a ocorrer. Hoje em dia, cerca de cem companhias de grande porte são responsáveis por cerca de $90 \%$ dos produtos farmacêuticos para consumo humano.

Por sua vez, $75 \%$ dessa produção é consumida, principalmente, nos Estados Unidos, Japão, Alemanha, França, Itália e Reino Unido 7. Nesses países, a proporção maior dos gastos com $\mathrm{P} \& \mathrm{D}$ tem sido direcionada ao atendimento de demandas de suas populações, por exemplo, doenças crônico-degenerativas ou proble- mas como calvície, enxaqueca, depressão, substâncias que, eventualmente, atuariam no adiamento do envelhecimento ou no controle da obesidade. Inversamente, países em desenvolvimento sofrem com a ausência de medicamentos específicos, sobretudo para as doenças denominadas negligenciadas. Sobre o tratamento dessa questão, a organização internacional humanitária Médicos Sem Fronteiras (MSF), entre outras, vem apontando a necessidade de institucionalizar mecanismos que levem os produtores a desenvolver pesquisas para o tratamento de doenças próprias da realidade sanitária dos países pobres. Para isso, é imprescindível a intervenção do Estado no fomento e na gestão em $P \& D$ de novos medicamentos eficazes e seguros para doenças que atingem ou ameaçam milhões de pessoas na África e América Latina. Essa organização 7 demonstra que, nos últimos cinco anos, nenhuma das vinte empresas farmacêuticas de maior faturamento bruto mundial lançou, no mercado, um único medicamento para qualquer uma das doenças negligenciadas e assinala, ainda, que o setor público também falhou na adoção de uma política que favorecesse o desenvolvimento de medicamentos destinados às necessidades epidemiológicas dessas sociedades, recomendando enfim que se direcionem recursos públicos para esses objetivos.

Denúncias de superfaturamento de matérias-primas e de abusos nos preços de medicamentos têm colocado, em evidência, o papel regulador do Estado frente a esse segmento industrial. A atuação do Estado se justifica, também, pela existência de falhas de mercado no setor, que justificariam a necessidade de uma estrutura de regulação voltada para amenizar os seus efeitos negativos sobre a população. Dentre as principais características que justificariam uma intervenção do Estado, podemos assinalar as seguintes: mercados concentrados, elevadas barreiras à entrada, demanda inelástica, variações nos preços dos produtos e assimetria de informações 4,8,9.

Para Bennett et al. 10, o Estado deveria ter uma atuação mais forte frente à indústria farmacêutica. Para tanto, seria necessário contar com linhas de financiamento para a aquisição e o acesso a medicamentos essenciais e campanhas de promoção do uso racional. Além disso, caberia estabelecer normas para a produção, registro, comercialização, distribuição e dispensação de medicamentos. Também, cabe uma ação continuada na formação e atualização de recursos humanos que lidam com a dispensação e a prescrição de medicamentos, em farmacoterapia, isenta do viés comercial hegemônico. 
Dentro desse contexto mais amplo, a produção e o mercado de medicamentos no Brasil adquirem características dignas de nota.

Em primeiro lugar, cabe mencionar que o Brasil situa-se entre os dez maiores mercados consumidores de produtos farmacêuticos do mundo, com faturamento anual que gira em torno de 10,3 bilhões de dólares 9 . Esse aspecto singulariza o Brasil das demais nações em desenvolvimento e faz despertar o interesse das empresas transnacionais pelo domínio desse vasto mercado.

Acompanhando a tendência internacional, o mercado brasileiro tem características oligopólicas, em que chamam a atenção a concentração por classes terapêuticas e o domínio de empresas transnacionais. Assim, observa-se que as atividades de produção, desenvolvimento e comercialização de medicamentos têm sido realizadas, principalmente, por companhias farmacêuticas transnacionais, geralmente de grande porte, com estrutura integrada com sua base mundial 1,8. Essa tendência observada no Brasil também pode ser encontrada em outros países do mundo.

Além disso, existem, aproximadamente, quatrocentas empresas farmacêuticas no Brasil. Dessas, vinte multinacionais dominam cerca de $80 \%$ do mercado, enquanto as 380 empresas de capital nacional são responsáveis por, aproximadamente, $20 \%$ do faturamento total. Dentre as empresas nacionais, destaca-se a Aché, que se mantém entre as dez maiores do setor 1,8 . Cabe salientar que o parque industrial brasileiro de medicamentos é bastante desenvolvido com relação à capacidade de fabricação de produtos finais. Ao mesmo tempo em que se constata esse desenvolvimento na produção, evidencia-se forte dependência da importação de farmacoquímicos. O desmantelamento de parte significativa do complexo industrial de química fina do Brasil promoveu o aumento da dependência da importação de matérias-primas pela indústria farmacêutica. Em 1997, o segmento industrial químico apresentava um faturamento anual de 42 bilhões de dólares, representando cerca de $15,7 \%$ da indústria de transformação, ou 3,6\% do PIB. Entretanto, o déficit comercial químico que, em 1990, era da ordem de US\$1,3 bilhão cresceu para U $\$$ \$ bilhões, oito anos mais tarde. "Esse período se traduziu no fechamento de mais de 1.000 unidades produtivas e no cancelamento de cerca de 500 projetos já definidos para o complexo industrial de química fina" 12 (p. 31).

$\mathrm{O}$ investimento em $\mathrm{P} \& \mathrm{D}$ para a produção de medicamentos em nosso país também é irrelevante. Apesar da excelente margem de lu- cro, não tem havido investimento tecnológico em empresas, estrangeiras ou nacionais, públicas ou privadas, instaladas no país 11 . Segundo o diagnóstico setorial de Hasenclever 1, as empresas do setor farmacêutico gastaram, em 1998, apenas $0,53 \%$ de seu faturamento em atividades de $\mathrm{P} \& \mathrm{D}$, enquanto a média de todas as empresas, nos demais setores, foi de 1,33\%.

Uma característica peculiar da indústria farmacêutica no Brasil é a existência de um parque público de laboratórios, de abrangência nacional, voltado para a produção de medicamentos primordialmente destinados aos programas de saúde pública em assistência farmacêutica. De uma maneira geral, o conjunto de laboratórios públicos é capaz de produzir, aproximadamente, 11 bilhões de unidades farmacêuticas/ano, com 195 apresentações farmacêuticas, abrangendo mais de 107 princípios ativos 12,13. A produção desses laboratórios representa cerca de $3 \%$ da produção nacional em valor e $10 \%$ em volume equivalente a cerca de $10 \%$ do total de compras em medicamentos do Ministério da Saúde (MS) 13 (Tabela 1).

Analisando a Tabela 1, podemos constatar que praticamente todas as Unidades da Federação das regiões Nordeste, Sudeste e Sul têm, pelo menos, um Laboratório Público de Produção de Medicamentos. Por outro lado, as regiões Centro-Oeste e Norte são desprovidas do mesmo complexo industrial farmacêutico. A exceção merece ser dada ao laboratório goiano. O laboratório de Manaus, vinculado à Universidade Federal do Amazonas, encontra-se em fase final de instalação. Por outro lado, podemos observar uma nítida predominância de laboratórios vinculados aos governos estaduais (9).

Bermudez 6,14,15 argumenta que, apesar de a dependência externa em matéria de medicamentos ser um problema comum aos países em desenvolvimento, o Brasil é um dos poucos países a possuir um parque público estatal de produção de medicamentos, instalado em várias regiões do território nacional. Constitui, portanto, um importante patrimônio público formado por 18 laboratórios, alguns com quase duzentos anos de fundação, os quais vêm contribuindo no abastecimento de medicamentos ao setor público, especialmente daqueles destinados a importantes doenças endêmicas que afligem a população, sem grande interesse comercial para o setor privado. Para esse autor, seu fortalecimento e consolidação são vistos como instrumento efetivo de apoio às ações governamentais e uma referência para a regulação do mercado nacional. No seu entender, todas as ações relacionadas com a produção e o abastecimento de medicamentos envolvem 
Laboratórios farmacêuticos oficiais. Brasil, 2003.

\begin{tabular}{|c|c|c|c|c|}
\hline Laboratório & UF & $\begin{array}{l}\text { Ano de } \\
\text { fundação }\end{array}$ & Personalidade jurídica & Produção* \\
\hline $\begin{array}{l}\text { Laboratório de Tecnologia Farmacêutica (LTF), } \\
\text { Universidade Federal da Paraíba }\end{array}$ & Paraíba & - & Autarquia & $193.080,0$ \\
\hline $\begin{array}{l}\text { Faculdade de Farmácia, Odontologia e Enfermagem } \\
\text { (FFOE), Universidade Federal do Ceará }\end{array}$ & Ceará & - & Autarquia & $7.200,0$ \\
\hline $\begin{array}{l}\text { Laboratório Químico Farmacêutico do Exército (LQFE), } \\
\text { Ministério do Exército }\end{array}$ & Rio de Janeiro & 1808 & Administração direta & $209.419,4$ \\
\hline Laboratório Farmacêutico da Marinha (LFM), Ministério da Marinha & Rio de Janeiro & 1906 & Administração direta & $120.800,0$ \\
\hline Fundação Ezequiel Dias (FUNED), Secretaria de Estado de Saúde & Minas Gerais & 1907 & $\begin{array}{l}\text { Fundação pública de } \\
\text { direito público }\end{array}$ & $692.340,0$ \\
\hline Instituto Vital Brazil S.A. (IVB), Secretaria de Estado de Saúde & Rio de Janeiro & 1918 & $\begin{array}{l}\text { Sociedade de economia } \\
\text { mista }\end{array}$ & $10.680,0$ \\
\hline $\begin{array}{l}\text { Instituto de Tecnologia de Fármacos (FAR-MANGUINHOS), } \\
\text { Fundação Oswaldo Cruz }\end{array}$ & Rio de Janeiro & 1956 & Unidade técnica & $1.289 .067,3$ \\
\hline $\begin{array}{l}\text { Indústria Química do Estado de Goiás (IQUEGO), } \\
\text { Secretaria de Estado de Saúde }\end{array}$ & Goiás & 1964 & $\begin{array}{l}\text { Sociedade de economia } \\
\text { mista }\end{array}$ & $618.000,0$ \\
\hline $\begin{array}{l}\text { Laboratório Farmacêutico do Estado de Pernambuco S.A. } \\
\text { (LAFEPE), Secretaria de Estado de Saúde }\end{array}$ & Pernambuco & 1967 & $\begin{array}{l}\text { Sociedade de economia } \\
\text { mista }\end{array}$ & $1.345 .680,0$ \\
\hline $\begin{array}{l}\text { Laboratório de Ensino, Pesquisa e Extensão em Medicamentos } \\
\text { e Cosméticos (LEPMC), Universidade Estadual de Maringá }\end{array}$ & Paraná & 1967 & Departamento & $21.000,0$ \\
\hline $\begin{array}{l}\text { Laboratório Farmacêutico de Santa Catarina (LAFESC), } \\
\text { Secretaria de Estado de Saúde }\end{array}$ & Santa Catarina & 1969 & Diretoria & $38.400,0$ \\
\hline $\begin{array}{l}\text { Laboratório Químico Farmacêutico da Aeronáutica (LAQFA), } \\
\text { Ministério da Aeronáutica }\end{array}$ & Rio de Janeiro & 1971 & Administração direta & $242.352,0$ \\
\hline $\begin{array}{l}\text { Fundação para o Remédio Popular (FURP), } \\
\text { Secretaria de Estado de Saúde }\end{array}$ & São Paulo & 1972 & $\begin{array}{l}\text { Fundação pública de } \\
\text { direito público }\end{array}$ & $3.903 .840,5$ \\
\hline $\begin{array}{l}\text { Laboratório Farmacêutico do Rio Grande do Sul (LAFERGS), } \\
\text { Fundação Estadual de Produção e Pesquisa em Saúde }\end{array}$ & Rio Grande do Sul & 1972 & Departamento & $375.800,0$ \\
\hline $\begin{array}{l}\text { Laboratório Industrial Farmacêutico de Alagoas (LIFAL), } \\
\text { Secretaria de Estado de Saúde }\end{array}$ & Alagoas & 1974 & $\begin{array}{l}\text { Sociedade de economia } \\
\text { mista }\end{array}$ & $1.728 .144,0$ \\
\hline $\begin{array}{l}\text { Laboratório de Produção de Medicamentos (LPM), } \\
\text { Universidade Estadual de Londrina }\end{array}$ & Paraná & 1989 & Autarquia especial & $96.000,0$ \\
\hline $\begin{array}{l}\text { Núcleo de Pesquisa em Alimentos e Medicamentos (NUPLAN), } \\
\text { Universidade Federal do Rio Grande do Norte }\end{array}$ & $\begin{array}{l}\text { Rio Grande } \\
\text { do Norte }\end{array}$ & 1991 & $\begin{array}{l}\text { Órgão suplementar } \\
\text { da UFRN }\end{array}$ & 876,3 \\
\hline $\begin{array}{l}\text { Laboratório Industrial Farmacêutico do Estado da Paraíba } \\
\text { (LIFESA), Secretaria de Estado de Saúde }\end{array}$ & Paraíba & 1997 & $\begin{array}{l}\text { Sociedade de economia } \\
\text { mista }\end{array}$ & $80.000,0$ \\
\hline Total & & & & 10. $972.679,2$ \\
\hline
\end{tabular}

* Em milhões de unidades farmacêuticas/ano.

Fonte: Associação dos Laboratórios Farmacêuticos Oficiais do Brasil 16.

aspectos da política industrial, científica, tecnológica e de saúde. Assim, e dada essa característica de envolver diferentes setores, as políticas públicas do setor de medicamentos devem, para ele, ser desenvolvidas no âmbito de diferentes ministérios.

Uma outra peculiaridade do processo brasileiro é o lugar que o Estado vem desempenhando no setor farmacêutico. Nesse caso, merece destaque a atividade regulatória, envolvendo o acompanhamento e o estabelecimento de limites para os preços praticados pelas empresas privadas pari passu o fortalecimento dos produtores oficiais, de modo a oferecer medicamentos a preços mais baixos e a reduzir os custos dos programas públicos de saúde. Além disso, a produção de medicamentos pela rede pública pode contribuir para minorar problemas no suprimento de determinados medicamentos, sobretudo daqueles de menor interesse para o setor privado. Os efeitos dessa política se fizeram sentir rapidamente. Far-Manguinhos, laboratório vinculado ao MS, por exemplo, aumentou dez vezes seu faturamento, passando de um patamar de US\$ 5 milhões para US\$ 50 milhões na década de 1990 4. Uma outra conseqüência do aumento da produção pública pode ser percebida com a redução dos pre- 
ços de medicamentos, particularmente com os anti-retrovirais.

Apesar do potencial de produção dos laboratórios oficiais, alguns problemas são identificados. Merecem destaque aqueles de ordem gerencial e administrativa. De acordo com a Associação dos Laboratórios Farmacêuticos Oficiais do Brasil (ALFOB) 16, os laboratórios se ressentem da falta de flexibilidade no processo de compras e da rigidez e deficiência na contratação e qualificação de pessoal. Esses fatores teriam repercussão na capacidade de resposta desse setor às demandas do mercado e do setor público.

Em suma, o panorama da produção de medicamentos no Brasil é dominado pela indústria transnacional, que, apesar da alta margem de lucro, praticamente não investe em P\&D. Além disso, os preços dos produtos dificultam o acesso da maioria da população ao seu consumo. Quanto à produção nacional, embora residual, nela se destacam os laboratórios oficiais por serem responsáveis por cerca de $75 \%$ das unidades dispensadas no SUS, no Programa de Assistência Farmacêutica Básica 13. Esse fato faz com que o Brasil se destaque entre os países em desenvolvimento.

\section{A Política Nacional de Medicamentos: regulamentação recente}

A história recente da elaboração e implementação de uma política pública de medicamentos no Brasil tem alguns marcos dignos de nota. Bermudez 6 destaca o pioneirismo do Brasil na formulação de políticas de medicamentos essenciais ao estabelecer, desde 1964, a denominada Relação Básica e Prioritária de Produtos Biológicos e Matérias para uso Farmacêutico Humano e Veterinário (Decreto n. 53.612/1964). Na década de 70, são promulgadas, ainda, a $L e i$ n. 5.991, de 17 de dezembro de 1973, regulamentada pelo Decreto $n$. 74.170 , de 10 de junho de 1974, que dispõe sobre o controle sanitário do comércio de drogas, medicamentos, insumos farmacêuticos e correlatos, e também a Lei n. 6.360, de 23 de setembro de 1976, regulamentada pelo Decreto $n$. 79.094, de 5 de janeiro de 1977, que dispõe sobre a vigilância sanitária a que ficam sujeitos os medicamentos, as drogas, os insumos farmacêuticos e correlatos, cosméticos, saneamentos e outros produtos. Entretanto, a criação da Central de Medicamentos (CEME), por meio do Decreto n. 68.806 de 1971, é considerada uma importante iniciativa do governo brasileiro em termos de planejamento, organização e aquisição de medica- mentos, de forma centralizada para todo o país. Dentre as atribuições da CEME, destacavam-se aquelas voltadas à assistência farmacêutica pública e ao abastecimento de medicamentos essenciais à população. Sua atuação se dava de forma coordenada nos campos da pesquisa e incentivo do desenvolvimento de fármacos, produção, padronização, aquisição e distribuição de medicamentos 17. Outro marco importante na regulamentação do setor foi o estabelecimento, em 30 de julho de 1973, por meio do Decreto $n$. 72.552, do primeiro Plano Diretor de Medicamentos, que considerou a racionalização da área mediante o Sistema Oficial de Produção de Medicamentos. Em 1976, é homologada a Relação Nacional de Medicamentos Básicos (RMB) por meio da Portaria MPAS/GM 514, de 18 de outubro de 1976. A RMB, aprovada pelo Conselho Diretor da CEME (Resolução n. 92 de 29 de setembro de 1976), era constituída de 300 substâncias, em 535 apresentações. A RMB, que foi posteriormente atualizada em 1977 (Portaria MPAS/ GM 817/77), passou a ser oficialmente denominada Relação Nacional de Medicamentos Essenciais (RENAME) pela Portaria Interministerial MPAS/MS/MEC 03, de 15 de dezembro de 1982 14, 18,19,20,21.

Outras iniciativas importantes da CEME foram o lançamento do Programa de Farmácia Básica (1987), que constava de uma seleção de aproximadamente 40 itens de medicamentos integrantes da RENAME, destinados ao uso ambulatorial (atenção primária), e o estabelecimento da Comissão Multidisciplinar de Revisão da RENAME (Portaria CEME 45, de 14 de novembro de 1996). Em 1997, o Governo Federal desativou a CEME, através da Medida Provisória 1.576, do Decreto n. 2.283 e da Portaria GM 1.085 17,18. Suas competências, planos e programas foram sendo assumidos, aos poucos, por várias instâncias do MS e também pelos estados e municípios.

Após a extinção da CEME, em meio a uma série de denúncias de desvio dos objetivos iniciais, um outro avanço verificado foi a promulgação, em 12 de outubro de 1998, da Política Nacional de Medicamentos (PNM) por meio da Portaria n. 3.916/MS. Com ela, o MS buscava ampliar e diversificar os serviços prestados no âmbito do SUS, atender demandas derivadas, entre outras razões, pelo envelhecimento populacional e pressões da sociedade civil pelo cumprimento do dispositivo constitucional que assegura o direito universal à saúde 9 .

A PNM é considerada o primeiro posicionamento formal e abrangente do governo brasileiro sobre a questão dos medicamentos no contexto da reforma sanitária. Foi formulada 
com base nas diretrizes da Organização Mundial da Saúde e expressa as principais diretrizes para o setor com o propósito de garantir a necessária segurança, eficácia e qualidade desses produtos, a promoção do uso racional e o acesso da população àqueles considerados essenciais 22. Entre suas orientações, constam, ainda, a adoção e revisão da RENAME, a regulamentação sanitária de medicamentos, o desenvolvimento científico e tecnológico, o desenvolvimento e capacitação de recursos humanos e a reorientação da assistência farmacêutica. Nesse sentido, a PNM prevê a articulação de um conjunto de atividades que envolvem desde o desenvolvimento de recursos humanos e tecnológicos até a promoção do acesso da população aos medicamentos essenciais.

A PNM foi acompanhada da revisão da RENAME em 1999, 15 anos após a sua primeira versão, e com nova atualização em 2002 por meio da Portaria n. 597/99 23. A partir de 1999, a RENAME passou a servir de referência para o direcionamento da produção farmacêutica e para a definição de listas de medicamentos essenciais nas esferas estaduais e municipais, conforme o perfil epidemiológico local. A RENAME representa um importante instrumento de racionalização da política de compras diretas do Governo Federal e fortalecimento dos programas estratégicos, bem como compras de estados e municípios no âmbito do Piso de Atenção Farmacêutica Básica do SUS. Em 1999, foi também criada a Agência Nacional de Vigilância Sanitária (ANVISA), que, com relação aos laboratórios oficiais, assumiu o registro de medicamentos, anteriormente, uma atribuição da CEME. Cabe ressaltar que, desde sua constituição, a ANVISA vem desempenhando um importante papel na fiscalização das condições de fabricação dos medicamentos, contribuindo para a adequação do parque público às boas práticas de produção.

No biênio 1999/2000, o tema "medicamentos" volta a suscitar o interesse da mídia e da sociedade em geral, culminando com a instalação, em novembro de 1999, de uma Comissão Parlamentar de Inquérito sobre Medicamentos (CPI) na Câmara dos Deputados 24 . As razões que justificaram sua instalação estão diretamente relacionadas com os aumentos de preços, perpetrados pelos produtores, para além dos índices inflacionários e com as questões suscitadas pela falsificação de produtos. As denúncias de adulteração de medicamentos de laboratórios internacionais privados com filiais no país feriram a imagem desse setor produtivo. Os casos do anticoncepcional Microvlar e do Androcur para câncer de próstata, ambos da Schering do Brasil, foram amplamente noticiados pela imprensa 11 .

$\mathrm{O}$ relatório da CPI dos medicamentos é um documento central para a análise das visões predominantes em diferentes segmentos envolvidos com pesquisa, produção, controle e consumo de medicamentos, merecendo destaque o Título $V$. Nele, são abordados os potenciais da produção públicos, tanto como agente regulador de mercado quanto pelo volume de compras da União, dos estados e dos municípios, estimado em $30 \%$ do mercado de medicamentos no país. É ressaltada a responsabilidade dos laboratórios públicos de se constituírem como produtores de medicamentos essenciais e como referência de preços, custos e qualidade de produção. Propõe que, no caso de compras aos laboratórios privados, os preços dos medicamentos equivalentes não devem ser superiores aos dos laboratórios oficiais 24 .

O diagnóstico e principais proposições da CPI sobre a produção pública de medicamentos identificam variados problemas; dentre os quais, a diluição do poder de compra do MS. De fato, nos últimos anos, o poder de compra institucional do Governo Federal se fragmentou, apesar de o MS ter permanecido na posição de grande comprador de medicamentos. Essa diluição teria sido conseqüência do deslocamento dos serviços e responsabilidades sanitárias para os estados e municípios em decorrência da implantação do SUS. Um outro aspecto assinalado no documento diz respeito à perda da capacidade de articulação nacional dos laboratórios públicos e ao direcionamento de sua produção para as necessidades do país como um todo. Com o fim da CEME e a deflagração do processo de descentralização do SUS, a atuação dos laboratórios voltou-se, prioritariamente, para as necessidades estaduais.

\section{Ausência de mecanismos de articulação e coordenação entre gestores}

Com relação a essa questão, o relatório da CPI propôs uma coordenação nacional de produção pública de fármacos e medicamentos, com a participação do MS, do Ministério da Ciência e Tecnologia (MCT), do Ministério da Educação, dos laboratórios produtores, dos Centros de Desenvolvimento Tecnológico e dos gestores estaduais e municipais de saúde.

\section{Natureza jurídica dos laboratórios}

Os laboratórios públicos de administração direta sofrem com a falta de flexibilidade e agilidade gerenciais, especialmente no que se refe- 
re à aquisição de insumos, comprometendo, muitas vezes, os prazos de entrega ou o engajamento dos laboratórios em compromissos de vulto com quantidades preestabelecidas. Nesse caso, o relatório recomendou a reestruturação e modernização institucional, gerencial, administrativa e técnica dos laboratórios, bem como a adoção de contratos de gestão e sistemas de gestão de qualidade total, de processos e produtos, nos moldes das empresas privadas.

\section{Falta de investimentos em P\&D}

Esse fato concorre para que os laboratórios oficiais sejam reféns dos grandes laboratórios internacionais, em um mercado orientado pela competitividade na inovação tecnológica. O relatório propõe a associação entre investimentos permanentes para dinamizar a pesquisa e o desenvolvimento tecnológico, com a promoção e atualização tecnológica dos laboratórios públicos.

\section{O papel dos laboratórios oficiais}

O relatório considerou que os laboratórios oficiais podem contribuir para aumentar a concorrência no setor e, sobretudo, facilitar o acesso das pessoas de baixa renda aos medicamentos, em especial aos de uso contínuo. Para tanto, devem funcionar com agilidade e nos mesmos padrões de eficiência da empresa privada, ou seja, sem as mazelas decorrentes do empreguismo e do excesso de burocracia, assegurando sempre a lealdade na concorrência 24 .

Um último ponto relevante neste trecho do relatório da CPI diz respeito às características dos medicamentos produzidos no Brasil e ao papel que os laboratórios estatais poderiam exercer. Ressalta o documento que a produção dos laboratórios privados brasileiros orientou sua política de produção de medicamentos segundo as leis de mercado, desconsiderando a especificidade e relevância dos medicamentos voltados para a população de baixa renda 24 . Com isso, eles acompanharam uma tendência internacional. Como mencionado anteriormente, o poder de compra tem sido o elemento definidor da agenda de prioridades de pesquisa e produção de medicamentos para as grandes empresas multinacionais. Muitas pesquisas são desenvolvidas, e novos produtos, formulados porque atendem à demanda dos grandes mercados consumidores localizados nos países desenvolvidos da América do Norte, Europa e Ásia. Essa lógica implica na falta de compromisso dessas empresas com a pesquisa e a produção de medicamentos que atendam às doen- ças que afetam, principalmente, as populações pobres, localizadas, sobretudo, na América do Sul e África. Com base em dados levantados nos últimos 25 anos, apenas 15 novas substâncias foram indicadas para doenças tropicais e tuberculose 7. Os laboratórios oficiais, que não operam segundo a lógica de mercado, poderiam cumprir uma das determinações do relatório da CPI, pesquisando e produzindo os medicamentos essenciais e aqueles presentes na RENAME.

Em resumo, a CPI reafirmou que o fortalecimento da produção pública constitui uma estratégia fundamental para reduzir os preços e eliminar a enorme distância entre oferta e demanda de medicamentos essenciais no Brasil e assim garantir à população usuária do SUS o acesso a esse insumo essencial para a saúde.

No âmbito do poder Executivo, a Secretaria de Gestão e Investimentos em Saúde, do MS, elaborou, em 2000, o Projeto de Modernização da Produção Pública de Medicamentos, que detalha e complementa aspectos presentes na PNM, faz um diagnóstico do setor, defende o fortalecimento da produção oficial e faz previsões de investimentos visando à modernização da produção pública de medicamentos 25 . Propõe, também, que os laboratórios oficiais aumentem a oferta de medicamentos essenciais, de uso contínuo, a preços reduzidos e regulem preços, custos e qualidade dos produtos.

O projeto envolvia a criação de uma coordenação colegiada dos processos públicos de produção, com vistas à compatibilização entre a demanda por medicamentos e a produção. Essa função seria desempenhada por um Conselho Estratégico de Produção, que seria uma estrutura supra-organizacional, composta por representantes de cada um dos laboratórios integrantes do programa de modernização da produção pública de medicamentos do MS e de compradores públicos.

Quanto ao financiamento do projeto, previa-se um montante total de investimento da ordem de US\$26,3 milhões, sendo US\$ 18,2 milhões provenientes do Banco Mundial e US\$ 8,1 milhões, de contrapartida das Unidades Federadas participantes 25 . Esse documento pressupunha, ainda, uma etapa inicial de reorganização da produção de medicamentos e etapas seguintes destinadas à ampliação do número de laboratórios participantes, à diversificação da linha de produção, à ampliação do número de fármacos produzidos, ao desenvolvimento tecnológico e ao aumento de qualidade. Todavia, tal proposta, apesar de sua importância e do avanço que significaria para a produção oficial, não foi adiante. 
Um outro marco importante nesse processo foi o diagnóstico do setor público de produção de medicamentos, realizado em julho de 2003, sob o patrocínio da Secretaria de Ciência e Tecnologia e Insumos Estratégicos (SCTIE) do MS e da ALFOB. O evento constituiu uma primeira reunião da área para aprimorar o diagnóstico de situação sobre o setor e subsidiar a formulação de políticas públicas nesse campo 13. Fruto dessa reunião foi elaborado um relatório subdividido em três temas:

a) A disponibilização de medicamentos como fator limitante na implementação de políticas públicas de saúde. Nesse sentido, propôs: (i) recuperar e ampliar os serviços de assistência farmacêutica na rede pública de saúde, nos diferentes níveis de atenção, qualificar os serviços existentes, descentralizar as ações e estabelecer mecanismos para avaliar custo/efetividade de programas, produtos, tecnologias e procedimentos em saúde; (ii) utilizar a RENAME enquanto instrumento racionalizador; e (iii) modernizar e ampliar a capacidade instalada de produção dos laboratórios oficiais com vistas ao suprimento do SUS e ao cumprimento do seu papel como referência de custo e qualidade da produção de medicamentos.

b) Os laboratórios farmacêuticos oficiais: diagnóstico preliminar de situação. A ALFOB salienta o potencial de produção dos laboratórios oficiais (estimada em 11 bilhões de unidades farmacêuticas/ano) e a importância do seu direcionamento ao atendimento das necessidades de medicamentos essenciais, com destaque para a atenção básica de saúde, a superação das restrições relativas à constituição jurídica e administrativa dos laboratórios e a renovação das instalações físicas e equipamentos, condizentes com as inovações tecnológicas do setor.

c) Organização do setor produtivo oficial. Organizar os laboratórios públicos em rede a ser formada pela adesão das instituições, conforme um protocolo, com coordenação articulada, envolvendo representações do MS, dos laboratórios oficiais, do Conselho Nacional de Secretários Municipais de Saúde (CONASEMS), do Conselho Nacional de Secretários de Saúde (CONASS) e da ANVISA. A estruturação em rede seria relevante para o fortalecimento do bloco público de produção, para a organização e racionalização do sistema produtivo oficial com vistas ao atendimento das diretrizes do SUS. A rede deveria, ainda, dar suporte aos programas estratégicos, principalmente aqueles envolvendo produção exclusivamente institucional, a qual deveria incluir o fomento à pesquisa e o desenvolvimento tecnológico.
Os investimentos solicitados pelos laboratórios oficiais deveriam considerar, também, verbas para a qualificação e capacitação de recursos humanos, em consonância com a necessidade de qualificação de linhas de produção e melhoria do processo de gestão. Dessa forma, a alocação de recursos passaria a obedecer a critérios rigorosos, estabelecidos a partir de análise consistente da capacidade de resposta de cada um dos laboratórios, conforme as diferentes realidades e capacidades de execução orçamentária e a apresentação de projetos.

Em geral, o relatório do seminário de julho de 2003 define, como prioritárias, a reestruturação do sistema de qualidade, aquisição de equipamentos para estudos de estabilidade e estudos de equivalência farmacêutica e bioequivalência relativa; a revisão e otimização das formulações dos medicamentos produzidos e a modernização e/ou aquisição de novas instalações e equipamentos. Ele define ainda a ampliação da rede existente (Tabela 2).

Nas conclusões são consideradas, ainda, que as análises apresentadas pelo MS e pela ALFOB, embora preliminares, sinalizam para a gravidade do problema, que se expressa na ausência de planejamento e avaliação das ações, bem como de uma efetiva condução pelo Estado 13.

\section{Considerações finais}

O MS tem manifestado formalmente a importância de algumas diretrizes para a política de medicamentos. Um primeiro aspecto dessa política se manifesta através da ampliação do acesso aos medicamentos. Além da aprovação da Política Nacional de Assistência Farmacêutica (Resolução n. 338/2004), foram tomadas algumas medidas governamentais que consideramos importante destacar. A primeira delas foi a constituição da SCTIE/MS, que passou a ser a instância responsável pela formulação da política nacional de assistência farmacêutica; a criação da Câmara de Medicamentos (CAMED Lei n. 10.742 de 2003), voltada à regulação do mercado; a instalação do Fórum de Competitividade da Cadeia Produtiva Farmacêutica, instalado em maio de 2003, uma iniciativa do Ministério de Desenvolvimento, Indústria e Comércio Exterior (MDIC) em coordenação com o MS e MCT; e a aquisição de uma nova planta industrial para Far-Manguinhos, que irá ampliar substancialmente a capacidade de produção do setor público 26. Quanto aos recursos direcionados para a modernização dos laboratórios oficiais, o MS estima que R\$ 182 milhões foram investidos entre 2003 e 2005. Esses in- 
Distribuição regional dos laboratórios oficiais em fase de estruturação.

\begin{tabular}{|c|c|c|c|}
\hline Região & $\mathbf{n}$ & Laboratórios & UF \\
\hline Norte & 1 & Fundação Universidade do Amazonas (FUAM) & Amazonas \\
\hline \multirow[t]{4}{*}{ Nordeste } & 4 & Laboratório Farmacêutico de Sobral (LAFAS) & Ceará \\
\hline & & Núcleo de Tecnologia Farmacêutica (NTF), Universidade Federal do Piauí & Piauí \\
\hline & & Universidade Estadual de Feira de Santana (UEFSFARMA) & Bahia \\
\hline & & $\begin{array}{l}\text { Laboratório Análises Clínicas e Bromatologia da Universidade Federal } \\
\text { do Ceará (LACT) }\end{array}$ & Ceará \\
\hline Centro-Oeste & 1 & Laboratório Farmacêutico de Tocantins (FARMATINS) & Tocantins \\
\hline \multirow[t]{2}{*}{ Sudeste } & 2 & Laboratório Municipal de Manipulação e Fitoterapia - Itatiaia & Rio de Janeiro \\
\hline & & Laboratório Industrial Farmacêutico (UFE), Universidade de Alfenas & Minas Gerais \\
\hline
\end{tabular}

Fonte: Ministério da Saúde 13.

vestimentos destinaram-se, principalmente, às adequações à Resolução n. 134, de 13 de julho de 2001, da ANVISA, que determina a todos os estabelecimentos fabricantes de medicamentos o cumprimento das diretrizes estabelecidas no Regulamento Técnico das Boas Práticas de Fabricação. Parte desses recursos também foi direcionada à instalação de novas unidades produtivas no país 26 .

Cabe ressaltar que a organização dos laboratórios oficiais em rede foi formalizada, em 2005, por meio da Portaria MS 843 de 2 de junho de 2005, que cria a Rede Brasileira de Produção Pública de Medicamentos. Essa deverá ser formada pelos laboratórios farmacêuticos oficiais do Brasil, mediante adesão institucional, baseada em protocolo harmonizado e pactuado no âmbito do seu comitê gestor, coordenado pelo MS, com a seguinte composição: cinco representantes do MS, dois da ANVISA, três da ALFOB, dois do CONASS e dois do CONASEMS 26. Como vimos nesse tópico e com base na regulamentação recente do setor, desde 1998, quando foi formulada a PNM, uma série de estudos e propostas têm surgido em torno da produção oficial de medicamentos. Entretanto, apesar de investimentos recentes, pelo Governo Federal, voltados à dotação de novas instalações em grande parte desses laboratórios, verifica-se uma dispersão de recursos e um alto grau de descontinuidade no processo de capacitação do parque de laboratórios farmacêuticos oficiais, para que possam cumprir seu papel de forma plena e junto ao SUS e à população. Os estudos e os documentos oficiais sobre o setor público de produção de medicamentos no Brasil, examinados neste trabalho, apontam a importância de fortalecer a produção oficial em função do aumento da demanda por medicamentos. O crescimento dessa demanda tem sido provocado, entre outros fatores, pelo envelhecimento populacional, assim como pelo fenômeno da medicalização promovido pela difusão publicitária da idéia de que o consumo de medicamentos é um indicador de bons níveis de saúde 27,28. Esses fatores, associados à dificuldade de acesso da maioria dos brasileiros, devido ao baixo poder aquisitivo, têm colocado, na ordem do dia, a questão da produção pública de medicamentos.

No entanto, os diagnósticos sobre o parque de laboratórios públicos realizados até o momento revelam que, apesar dos problemas políticos, administrativos e tecnológicos que enfrentam, as empresas vêm encontrando formas de se revitalizarem a partir da modernização tecnológica, tanto com incentivos federais quanto com recursos próprios.

Recentemente, a produção pública de medicamentos adquiriu uma maior visibilidade em conseqüência da atividade regulatória desenvolvida no estabelecimento de limites aos preços de medicamentos para AIDS (anti-retrovirais), praticados por empresas multinacionais 28 .

Descortina-se, assim, um cenário próprio para estudos e pesquisas que contribuam para um maior e melhor conhecimento sobre a realidade e potencial dos laboratórios públicos produtores de medicamentos. 


\section{Resumo}

Este artigo analisa o papel da produção pública de medicamentos no Brasil, a partir de análise bibliográfica e documental. Examina as principais características da indústria farmacêutica e do mercado de medicamentos em suas dimensões internacional e nacional. Enfatiza, particularmente, a produção de medicamentos do setor público, bem como aspectos da visão governamental sobre o tema. O estudo se fundamenta na análise de documentos oficiais do Ministério da Saúde. A exposição conclui com um diagnóstico da situação atual do parque de laboratórios públicos, que mostra a existência de problemas de ordem política, administrativa e de funcionamento, aliados à baixa capacitação tecnológica e à escassez de recursos humanos qualificados em geral.

Preparações Farmacêuticas; Política Nacional de Medicamentos; Laboratório Oficial

\section{Referências}

1. Hasenclever L, organizador. Diagnóstico da indústria farmacêutica brasileira. Brasília: Organização das Nações Unidas para a Educação, a Ciência e a Cultura/Rio de Janeiro: Universidade Federal do Rio de Janeiro; 2002.

2. Frenkel J, Reis JA, Araújo Jr. JT, Naidin LC. Tecnologia e competição na indústria farmacêutica brasileira. Rio de Janeiro: Financiadora de Estudos e Projetos; 1978.

3. Oliveira MA. Tecnociência, ativismo e a política do tratamento da Aids [Tese de Doutorado]. Rio de Janeiro: Coordenação de Programas de Pósgraduação em Engenharia, Universidade Federal do Rio de Janeiro; 2001.

4. Gadelha CAG, Quental C, Fialho BC. Saúde e inovação: uma abordagem sistêmica das indústrias de saúde. Cad Saúde Pública 2003; 19:47-59.

5. MSH. Managing drug supply: the selection, procurement, distribution, and use of pharmaceuticals. Bloomfield: Kumarian Press: 1997.

6. Bermudez JAZ. Indústria farmacêutica, estado e sociedade - crítica da política de medicamentos no Brasil. São Paulo: Editora Hucitec/Rio de Janeiro: ABRASCO; 1995.

7. Médicos Sem Fronteiras. Desequilíbrio fatal: a crise em pesquisa e desenvolvimento de drogas para doenças negligenciadas. Geneva: Grupo de Trabalho de Drogas para Doenças Negligenciadas, Médicos Sem Fronteiras; 2001.

\section{Colaboradores}

E. A. Oliveira estruturou o conteúdo do texto, avaliou os dados e redigiu a versão final do artigo. M. E. Labra orientou a redação do artigo, revisou e implementou as modificações necessárias. J. Bermudez contribuiu na concepção da pesquisa e análise dos dados.
8. Gadelha CAG. Estudo de competitividade por cadeias integradas no Brasil: impactos das zonas de livre comércio. Cadeia: complexo da saúde. Nota técnica final. Campinas: Núcleo de Economia Industrial e da Tecnologia, Instituto de Economia, Universidade Estadual de Campinas/Ministério do Desenvolvimento, da Indústria e do Comércio Exterior/Ministério da Ciência e Tecnologia/Financiadora de Estudos e Projetos; 2002.

9. Bermudez JAZ, Epsztein R, Oliveira MA, Hasenclever L. O acordo Trips da OMC e a proteção patentária no Brasil: mudanças recentes e implicações para a produção local e o acesso da população aos medicamentos. Rio de Janeiro: Escola Nacional de Saúde Pública, Fundação Oswaldo Cruz/ Organização Mundial da Saúde; 2000.

10. Bennett S, Quick JD, Velásquez G. Public-private roles in the pharmaceutical sector: implications for equitable access and rational drug use. Geneva: World Health Organization; 1997. (Health Economics and Drugs, DAP Series, 5).

11. Callegari L. Análise setorial. A indústria farmacêutica. São Paulo: Gazeta Mercantil; 2000.

12. Pinheiro ES. Laboratórios farmacêuticos governamentais e o Sistema Único de Saúde. In: Bonfim JR, Mercucci VL, organizadores. A construção da política nacional de medicamentos. São Paulo: Editora Hucitec/Rio de Janeiro: ABRASCO; 1997. p. 81-6. 
13. Ministério da Saúde. Produção oficial de medicamentos: diagnóstico, limitações e perspectivas. Brasília: Secretaria de Ciência e Tecnologia e Insumos Estratégicos, Ministério da Saúde; 2003.

14. Bermudez JAZ. Produção de medicamentos no setor governamental e as necessidades do Sistema Único de Saúde. In: Bonfim JR, Mercucci VL, organizadores. A construção da política nacional de medicamentos. São Paulo: Editora Hucitec/ Rio de Janeiro: ABRASCO; 1997. p. 72-80.

15. Bermudez JAZ. Remédios: saúde ou indústria? A produção de medicamentos no Brasil. Rio de Janeiro: Relume-Dumará; 1992.

16. Associação dos Laboratórios Farmacêuticos Oficiais do Brasil. Os laboratórios farmacêuticos oficiais: diagnóstico preliminar de situação sob duas percepções. Seminário "Produção Oficial de Medicamentos: Diagnóstico, Limitações e Perspectivas". Brasília: Associação dos Laboratórios Farmacêuticos Oficiais do Brasil/Secretaria de Ciência, Tecnologia e Insumos Estratégicos, Ministério da Saúde; 2005.

17. Lucchesi G. Dependência e autonomia no setor farmacêutico: um estudo da CEME [Dissertação de Mestrado]. Rio de Janeiro: Escola Nacional de Saúde Pública, Fundação Oswaldo Cruz; 1991.

18. Costa EA, Rozenfeld S. Constituição da vigilância sanitária no Brasil. In: Rozenfeld S, organizadora. Fundamentos da vigilância sanitária. Rio de Janeiro: Editora Fiocruz; 2000. p. 15-60.

19. Cosendey MAE. Análise da implantação do programa farmácia básica: um estudo multicêntrico em cinco estados do Brasil [Tese de Doutorado]. Rio de Janeiro: Escola Nacional de Saúde Pública, Fundação Oswaldo Cruz; 2000.
20. Wilken PR, Bermudez JAZ. A farmácia hospitalar: como avaliar? Rio de Janeiro: Ágora da Ilha; 1999.

21. Silva RCS, Bermudez JAZ. Medicamentos excepcionais ou de alto custo no Brasil. In: Bermudez JAZ, Oliveira MA, Esher A, organizadores. Acesso a medicamentos: direito fundamental, papel do Estado. Rio de Janeiro: Escola Nacional de Saúde Pública, Fundação Oswaldo Cruz; 2004. p. 203-31.

22. Ministério da Saúde. Política nacional de medicamentos. Brasília: Secretaria de Políticas Públicas, Brasília: Ministério da Saúde; 2000.

23. Ministério da Saúde. Relação nacional de medicamentos essenciais - RENAME-2000. Brasília: Secretaria de Políticas de Saúde, Ministério da Saúde; 2000.

24. Câmara dos Deputados. Relatório da CPI - Medicamentos. Comissão Parlamentar de Inquérito destinada a investigar os reajustes de preços e a falsificação de medicamentos, materiais hospitalares e insumos de laboratórios. Brasília: Câmara dos Deputados; 2000.

25. Ministério da Saúde. Projeto de modernização da produção pública de medicamentos. Brasília: Secretaria de Gestão e Investimentos, Ministério da Saúde; 2000.

26. Departamento de Assistência Farmacêutica e Insumos Estratégicos, Secretaria de Ciência, Tecnologia e Insumos Estratégicos, Ministério da Saúde. A política de assistência farmacêutica: efetivando o acesso, a qualidade e a humanização da assistência farmacêutica, com controle social. http://dtr2001.saude.gov.br/sctie/portal_daf/index_daf.htm (acessado em 01/Ago/2005).

27. Lefevre F. O medicamento como mercadoria simbólica. São Paulo: Cortez Editora; 1991.

28. Barros JAC. Políticas farmacêuticas: a serviço dos interesses da saúde? Brasília: Organização das Nações Unidas para a Educação, a Ciência e a Cultura/Agência Nacional de Vigilância Sanitária; 2004.

Recebido em 24/Mai/2005

Versão final reapresentada em 01/Dez/2005

Aprovado em 19/Jan/2006 